TRANSACTIONS OF THE

AMERICAN MATHEMATICAL SOCIETY

Volume 366, Number 2, February 2014, Pages 1095-1117

S 0002-9947(2013)05948-X

Article electronically published on August 8, 2013

\title{
A CANTOR-MANDELBROT-SIERPIŃSKI TREE IN THE PARAMETER PLANE FOR RATIONAL MAPS
}

\author{
ROBERT L. DEVANEY
}

\begin{abstract}
In this paper we prove the existence of a Cantor-MandelbrotSierpiński tree (a CMS tree) in the parameter plane for the family of rational maps $z^{2}+\lambda / z^{2}$. This tree consists of a main trunk that is a Cantor necklace. Infinitely many Cantor necklaces branch off on either side of the main trunk, and between each of these branches is a copy of a Mandelbrot set.
\end{abstract}

In the family of rational maps given by

$$
F_{\lambda}(z)=z^{2}+\frac{\lambda}{z^{2}}
$$

there are usually three distinct components in both the dynamical plane and the parameter plane. In the dynamical plane, the first component is the immediate basin of attraction of the fixed point at $\infty$. The second component is the eventually escaping set, i.e., the preimages of the immediate basin of $\infty$ (all of which are disjoint from the immediate basin as long as the Julia set of $F_{\lambda}$ is not a Cantor set). The third component is the set of non-escaping points. This piece often (though not always) consists of three separate subpieces: the boundary curves of the escaping disks, a set of buried points, and an infinite collection of closed sets that are each homeomorphic to a Julia set of a given quadratic polynomial.

In a similar fashion, the parameter plane also breaks into three disjoint pieces. The first is the set of parameters for which the critical values of $F_{\lambda}$ lie in the immediate basin of $\infty$ and hence the Julia set is a Cantor set [6]. The second is the set of parameters $\lambda$ for which the critical orbits eventually escape to $\infty$. When this happens, it is known that the Julia set is a Sierpiński curve (i.e., a set homeomorphic to the Sierpiński carpet fractal) 6]. Such Julia sets are known to occur in many different families of complex maps (see, for example, 3, 17, 18, and 21]). It is known that, for our family, there are infinitely many disjoint regions in the parameter plane where this happens and that each of these regions is an open disk 20]. We call these regions in the parameter plane Sierpiński holes. The final possibility is that one and hence all of the free critical orbits do not escape to $\infty$. Here again there are three main subcases. The first is when the critical orbits lie on a boundary curve of the escaping set; the second is where the critical orbits lie on buried points in the Julia set; and the third is where we have quadratic-like components in the non-escaping set, in which case the parameter usually lies in

Received by the editors November 5, 2011 and, in revised form, August 17, 2012.

2010 Mathematics Subject Classification. Primary 37F10; Secondary 37F45.

This work was partially supported by grant \#208780 from the Simons Foundation.

(C)2013 American Mathematical Society 
a baby Mandelbrot set in the parameter plane. We shall show in this paper that there are infinitely many distinct copies of the Mandelbrot set in the parameter plane.

The parameter plane for the family $z^{2}+\lambda / z^{2}$ is displayed in Figure 1] The external grey region contains parameters for which the Julia set of $F_{\lambda}$ is a Cantor set. The bounded grey disks in this picture are the Sierpiński holes, and the black regions are (usually) Mandelbrot sets.

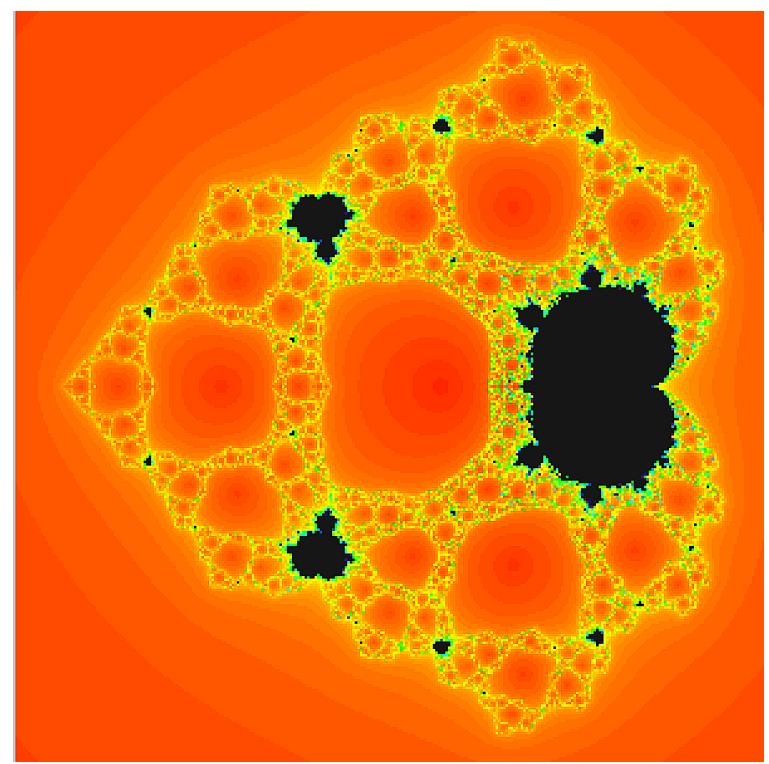

Figure 1. The parameter plane for $z^{2}+\lambda / z^{2}$.

At this time we still do not understand the complete structure of Mandelbrot sets, even in the case of quadratic polynomials. However, for our family, there is a complete classification of the dynamical behavior for parameters in the family that lie in the Sierpiński holes, and these Sierpiński holes make up a large portion of the entire parameter plane. Therefore the natural question is: how do these various regions in the parameter plane fit together?

Our goal in this paper is to give a partial answer to this question. We shall prove the existence of a Cantor-Mandelbrot-Sierpiński tree (a CMS tree) in the parameter plane. This object will detail the arrangement of infinitely many Mandelbrot sets, Sierpiński holes, and buried parameters in a way that begins to give a map of the entire structure of the parameter plane.

A CMS tree consists of a main "trunk" with infinitely many "branches" attached together, with infinitely many baby Mandelbrot sets also attached to the main trunk, one between each pair of branches. The trunk and the branches of the CMS tree are all "Cantor necklaces". A Cantor necklace is a set that is a continuous, one-to-one image of the Cantor middle-thirds necklace. The middle-thirds necklace is obtained by starting with the Cantor middle-thirds set along the real axis in the plane, and then adding in an open disk in place of each removed open middlethirds interval. In the parameter plane, the open disks in each Cantor necklace are 
Sierpiński holes and the non-endpoints in the Cantor set portion of the necklace are buried parameters.

In Figure 2 we display several of the Cantor necklaces in the CMS tree. The black lines run through the Cantor set portion of the necklace as well as all of the attached Sierpinski holes. The main trunk of the tree (the principal Cantor necklace) runs along the horizontal (negative real) axis. The Cantor necklace branches each emanate from certain Sierpiński holes in the trunk and extend up and down to the Cantor set regime in the parameter plane. From each Sierpiński hole in the main trunk from which a branch emanates, note that there is also a baby Mandelbrot set extending from this hole to the Cantor set regime, again from both above and below.

One of the main tools for understanding the structures in the dynamical and parameter planes involves external rays. For polynomials as well as for our family of maps, there are natural holomorphic maps that take the open unit disk in the plane univalently onto the immediate basin of $\infty$ in the dynamical plane (when the Julia set is connected), and to the external region in the parameter plane. The images of the straight rays under these maps are the external rays, and how they land on the Julia set or in the parameter plane determines quite a bit of the corresponding structure. In [2] and [9], it was shown how to extend these external rays into the Julia sets of certain rational maps, including those in our family. The construction of the Cantor necklaces in this paper provides a way to extend the external rays in the parameter plane into the interior of the interesting region in the parameter plane.

In this paper we concentrate primarily on the family $z^{2}+\lambda / z^{2}$. Many other recent papers have dealt more generally with the family $z^{n}+\lambda / z^{d}$ where $n, d \geq 2$. It turns out that the situation when both $n$ and $d$ are equal to 2 is much more complicated than the higher degree cases. There are a variety of reasons for this (see [10]). Perhaps the most important reason is that, as long as $n$ and $d$ are not both equal to 2, there is a McMullen domain surrounding the origin in the parameter plane. This is a punctured open disk of parameters around 0 for which the Julia set is always a Cantor set of simple closed curves [14. As a consequence, the structure of the parameter plane near 0 is quite simple in the higher degree cases. There is no such domain for $z^{2}+\lambda / z^{2}$ and, as a consequence, the structure of the parameter plane near 0 is much more complicated. In addition, many of the problems that we shall encounter in the construction of the CMS tree near the origin do not occur in the higher degree case. Finally, instead of having Cantor necklaces in the dynamical and parameter planes in the higher degree case, there are now Cantor webs (see [11]). These are very different types of objects.

\section{Preliminaries}

In this paper we shall concentrate on the specific family of maps given by

$$
F_{\lambda}(z)=z^{2}+\frac{\lambda}{z^{2}}
$$

where $\lambda \neq 0$. We begin by reviewing some basic properties of functions in this family. See [1] or [4 for proofs of these facts.

Note first that 0 is the only pole for each function in this family. The points $(-\lambda)^{1 / 4}$ are prepoles for $F_{\lambda}$ since they are mapped by $F_{\lambda}$ directly to 0 . The free critical points for $F_{\lambda}$ occur at the four points $\lambda^{1 / 4}$. Since $F_{\lambda}\left(\lambda^{1 / 4}\right)= \pm 2 \lambda^{1 / 2}= \pm v_{\lambda}$, 


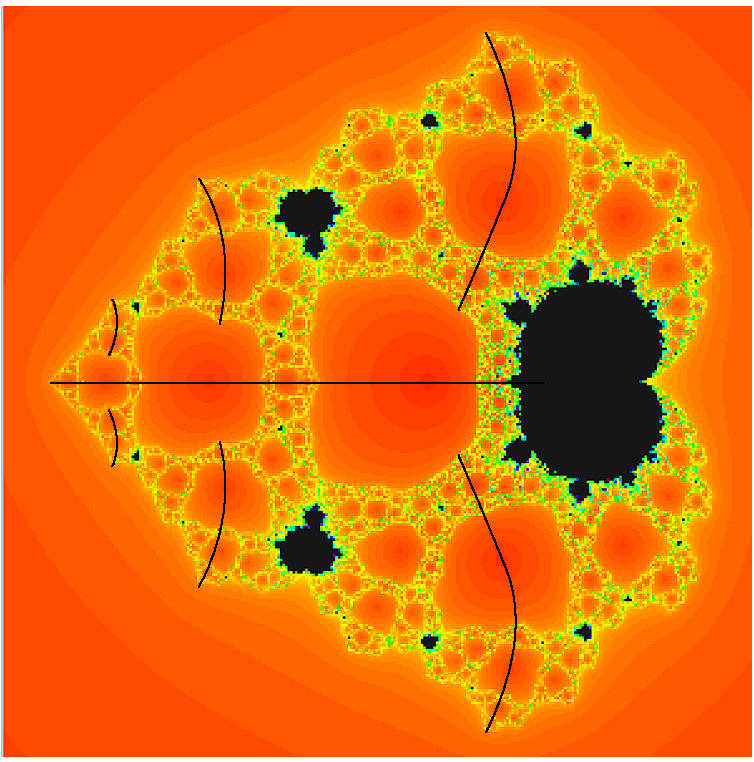

Figure 2. The parameter plane for $z^{2}+\lambda / z^{2}$.

there are only two critical values for $F_{\lambda}$. Also, $F_{\lambda}^{2}\left(\lambda^{1 / 4}\right)=1 / 4+4 \lambda$, so each of the four critical points lies on the same forward orbit after two iterations. Thus the orbit of $1 / 4+4 \lambda$ is the tail of the free critical orbit. The points at $\infty$ and 0 are also critical points, but they are not free since $\infty$ is fixed and 0 maps onto $\infty$.

The circle given by $|z|=\left|\lambda^{1 / 4}\right|$ is known as the critical circle for this family and is denoted by $C_{\lambda}$. A computation shows that the critical circle is mapped onto the straight line segment connecting the two critical values and passing through the origin. $F_{\lambda}$ takes $C_{\lambda}$ in four-to-one fashion onto this segment except at the critical values, each of which has only two preimages. More generally, $F_{\lambda}$ takes any circle centered at the origin two-to-one onto an ellipse whose foci are $\pm v_{\lambda}$.

The point at $\infty$ is a superattracting fixed point for $F_{\lambda}$ and we denote its immediate basin of attraction by $B_{\lambda}$. Let $\partial B_{\lambda}$ denote the boundary of $B_{\lambda}$. The basin $B_{\lambda}$ is a (forward) invariant set for $F_{\lambda}$ in the sense that, if $z \in B_{\lambda}$, then $F_{\lambda}^{n}(z) \in B_{\lambda}$ for all $n \geq 0$. The same is true for $\partial B_{\lambda}$.

Let $J=J\left(F_{\lambda}\right)$ be the Julia set of $F_{\lambda}$. By definition, $J\left(F_{\lambda}\right)$ is the set of points at which the family of iterates of $F_{\lambda}$ fails to be a normal family in the sense of Montel. Equivalently, $J\left(F_{\lambda}\right)$ is the closure of the set of repelling periodic points of $F_{\lambda}$ and it is also the boundary of the set of points whose orbits tend to $\infty$. See [16] for proofs of these equivalences.

The following was proved in [4].

Theorem. For the family of rational maps given by

$$
F_{\lambda}(z)=z^{2}+\frac{\lambda}{z^{2}}
$$

(1) If the critical values lie in $B_{\lambda}$, then $J\left(F_{\lambda}\right)$ is a Cantor set.

(2) Otherwise, $J\left(F_{\lambda}\right)$ is a compact, connected set and $B_{\lambda}$ is open and simply connected. 
(3) In particular, if the critical orbit escapes to $\infty$ but the critical values do not lie in $B_{\lambda}$, then $J\left(F_{\lambda}\right)$ is a Sierpinski curve, i.e., a set that is homeomorphic to the Sierpinski carpet fractal.

By the above theorem, the Julia set of $F_{\lambda}$ is either connected or totally disconnected. We call the set of parameters for which $J\left(F_{\lambda}\right)$ is connected the connectedness locus in the parameter plane; the complementary region is the Cantor set locus. In case (3) of this theorem, since the critical values do not lie in $B_{\lambda}$, it is known that the preimage of $B_{\lambda}$ surrounding the origin, $T_{\lambda}$, is disjoint from $B_{\lambda}$. The map is two-to-one on both $B_{\lambda}$ and $T_{\lambda}$. Since $F_{\lambda}$ has degree 4 , these two sets contain all of the preimages of points in $B_{\lambda}$. We thus call $T_{\lambda}$ the trap door, since any orbit that eventually enters $B_{\lambda}$ must do so by passing through $T_{\lambda}$.

Remark. For the families of maps

$$
F_{\lambda}(z)=z^{n}+\frac{\lambda}{z^{d}}
$$

where $n, d \geq 2$ but are not both equal to 2 , there is another possibility in the above theorem. In this case it is possible that both of the critical values lie in the trap door. Then it is known that the Julia set is a Cantor set of simple closed curves all surrounding the origin [14. However, this cannot occur when $n=2$. See 6 .

Each of the maps $F_{\lambda}$ possess certain symmetries. For example, we have that $F_{\lambda}(-z)=F_{\lambda}(z)$ and $F_{\lambda}(i z)=-F_{\lambda}(z)$ so that $F_{\lambda}^{2}(i z)=F_{\lambda}^{2}(z)$ for all $z \in \mathbb{C}$. As a consequence, each of the sets $B_{\lambda}, T_{\lambda}$, and $J\left(F_{\lambda}\right)$ are invariant under $z \mapsto i z$. We therefore say that these sets possess fourfold symmetry.

There is a second symmetry present for this family. Consider the map $H_{\lambda}(z)=$ $\sqrt{\lambda} / z$. Note that there are two such maps depending upon which square root of $\lambda$ we choose. $H_{\lambda}$ is an involution and we have $F_{\lambda}\left(H_{\lambda}(z)\right)=F_{\lambda}(z)$. As a consequence, $H_{\lambda}$ also preserves $J$. The involution $H_{\lambda}$ also preserves the circle of radius $|\lambda|^{1 / 4}$, the critical circle, and interchanges the interior and exterior of this circle. Hence $J$ is symmetric about the critical circle with respect to the action of $H_{\lambda}$. Also, $H_{\lambda}$ maps $B_{\lambda}$ to $T_{\lambda}$ univalently, and vice versa.

In analogy with the well-studied quadratic polynomial family $z \mapsto z^{2}+c$, since $F_{\lambda}$ has degree two on $B_{\lambda}$, it is known that $F_{\lambda}$ is conjugate to $z \mapsto z^{2}$ on $B_{\lambda}$ when the critical values do not lie in $B_{\lambda}$ (in case (2) of the above Theorem). That is, there is an analytic homeomorphism $\phi_{\lambda}: B_{\lambda} \rightarrow \mathbb{D}$ (the Böttcher coordinate) that satisfies $\phi_{\lambda} \circ F_{\lambda}(z)=\left(\phi_{\lambda}(z)\right)^{2}$ for all $z \in B_{\lambda}$. Here $\mathbb{D}$ is the open unit disk in $\mathbb{C}$. Since the map $z \mapsto z^{2}$ preserves the straight rays $\operatorname{Arg} z=$ constant, the inverse images of these straight rays under $\phi_{\lambda}$ are preserved by $F_{\lambda}$. These curves are known as external rays. We denote the external ray corresponding to the straight ray $\operatorname{Arg} z=2 \pi \theta$ by $\xi_{\theta}^{\lambda}$. We then parametrize this ray by setting $\xi_{\theta}^{\lambda}(t)=\phi_{\lambda}^{-1}(\exp (2 \pi i \theta t))$. In particular, there is an external ray $\xi_{0}^{\lambda}$ corresponding to the ray $\operatorname{Arg} z=0$. It is known (see [19]) that this ray limits on the point $p_{\lambda}$ in $\partial B_{\lambda}$ which is the unique fixed point in $\partial B_{\lambda}$. In addition, it is easy to check that $\xi_{0}^{\lambda}$ lies along the positive real axis when $\lambda$ is real. Similarly, the external ray $\xi_{1 / 2}^{\lambda}$ corresponding to $\operatorname{Arg} z=\pi$ limits on the point $-p_{\lambda} \in \partial B_{\lambda}$ and lies along the negative real axis when $\lambda$ is real.

\section{Cantor necklaces in the Dynamical Plane}

Our goal in this section is to construct a collection of Cantor necklaces in the dynamical plane. We begin by proving the existence of an invariant Cantor set 
in the dynamical plane. Then we connect the "endpoints" of this Cantor set with curves that lie in preimages of $B_{\lambda}$ to produce internal rays in the dynamical plane. Finally, we adjoin various preimages of $T_{\lambda}$ to produce a Cantor necklace. We first construct the principal Cantor necklace in the dynamical plane. We then pull this necklace back using various preimages of $F_{\lambda}$ to construct infinitely many other such necklaces.

2.1. Invariant Cantor sets. For the rest of this section we shall consider only parameters $\lambda$ for which $0<\operatorname{Arg} \lambda<2 \pi$ and $0<|\lambda|<1$. Call this open set in the parameter plane $\mathcal{P}$. For $\lambda \in \mathcal{P}$, we have the following escape criterion.

Proposition (The Escape Criterion). Suppose $|\lambda|<1$ and $|z| \geq 2$. Then $\left|F_{\lambda}(z)\right|>$ $|z|$, so $z \in B_{\lambda}$ and $J\left(F_{\lambda}\right)$ is contained in the open disk $|z|<2$.

Proof. If $|z| \geq 2$, then we have

$$
\left|F_{\lambda}(z)\right| \geq|z|^{2}-\frac{|\lambda|}{|z|^{2}} \geq 2|z|-\frac{1}{4}>\frac{3}{2}|z| .
$$

Inductively, we find

$$
\left|F_{\lambda}^{n}(z)\right| \geq\left(\frac{3}{2}\right)^{n}|z|
$$

Therefore the orbit of any such $z$ tends to $\infty$ and thus all points on or outside the circle of radius 2 lie in $B_{\lambda}$.

Recall that the critical points of $F_{\lambda}$ are given by $\lambda^{1 / 4}$. Therefore one of the critical points of $F_{\lambda}$, say $c_{\lambda}$, lies on the straight ray given by $t \exp (i \eta / 4)$ with $t>0$ where $\eta=\operatorname{Arg} \lambda$ and $0<\eta<2 \pi$, The critical point $-c_{\lambda}$ lies on the negative of this straight ray and $F_{\lambda}$ maps both of these rays two-to-one onto the portion of the straight line with argument $\theta=\eta / 2$ extending from the critical value $v_{\lambda}=2 \sqrt{\lambda}$ whose argument is $\eta / 2$ to $\infty$. Note that the image of each ray is disjoint from the ray itself since we have assumed that $0<\eta<2 \pi$. There is a third critical point, $-i c_{\lambda}$, of $F_{\lambda}$ lying on the ray with argument $\theta=\eta / 4-\pi / 2$. This ray (and its negative which contains the fourth critical point, $i c_{\lambda}$ ) is mapped in two-to-one fashion to the portion of the ray $\theta=-\eta / 2$ extending from $-v_{\lambda}=-2 \sqrt{\lambda}$ to $\infty$.

By the escape criterion, we know that any point on or outside $r=2$ is mapped closer to $\infty$. Let $\beta_{\lambda}$ denote the ellipse that is the image of the circle $r=2$, so that $\beta_{\lambda} \subset B_{\lambda}$. Using the involution $H_{\lambda}$, there is a second circle, namely $r=|\lambda|^{1 / 2} / 2$, that is also mapped two-to-one onto $\beta_{\lambda}$.

Consider the open region $I_{0}=I_{0}(\lambda)$ bounded by the rays $\theta=\eta / 4$ and $\theta=$ $\eta / 4-\pi / 2$ and the circular portions of the two preimages of $\beta_{\lambda}$ connecting these lines. The set $I_{0}$ is a quarter of an annulus. Let $I_{j}$ be the other quarter annuli indexed in the counterclockwise direction, so $I_{2}=-I_{0}$. We call $I_{0}$ (resp., $I_{2}$ ) the right (resp., left) fundamental sector. These two fundamental sectors are a pair of disjoint, open, simply connected regions in $\mathbb{C}$. See Figure 3 , Since $c_{\lambda}$ rotates one quarter of a turn as $\operatorname{Arg} \lambda$ rotates from 0 to $2 \pi$, we have that $I_{0}(\lambda)$ lies in the right half plane for all $\lambda \in \mathcal{P}$, whereas $I_{2}(\lambda)$ lies in the left half plane.

Proposition. $F_{\lambda}$ maps each of the two fundamental sectors in one-to-one fashion onto the open set $\mathcal{O}$ bounded by $\beta_{\lambda}$ minus the portions of the two straight lines $\theta= \pm \eta / 2$ extending from the critical values $\pm v_{\lambda}$ to $\beta_{\lambda}$. Therefore the image of each of these fundamental sectors contains the closures of both $I_{0}$ and $I_{2}$ in its interior. 


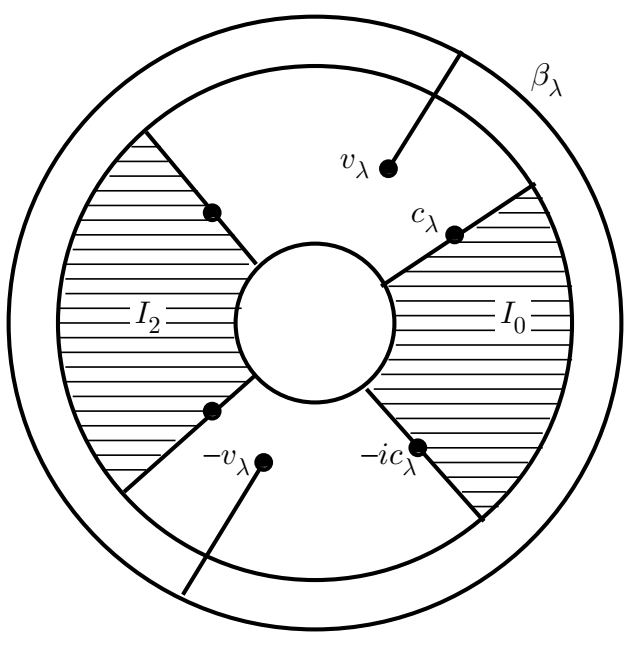

FiguRE 3. $I_{0}$ and $I_{2}$ and their image under $F_{\lambda}$, which is the interior of $\beta_{\lambda}$ minus the two segments connecting this circle to the critical values.

Proof. The images of the straight rays bounding $I_{0}$ and $I_{2}$ are contained in the rays $\theta= \pm \eta / 2$, both of which lie outside these sectors. The image of the outer circular boundary of each fundamental sector is a curve comprising exactly one-half of $\beta_{\lambda}$ connecting the two rays through $\pm v_{\lambda}$, while the inner boundary of each sector is mapped to the opposite half of $\beta_{\lambda}$. Hence each fundamental sector is mapped onto the open disk bounded by $\beta_{\lambda}$ minus the two portions of the rays $\theta= \pm \eta / 2$ lying beyond the critical values. This set is $\mathcal{O}$. By fourfold symmetry, this map is one-to-one on each fundamental sector.

Since $F_{\lambda}$ maps the union of the fundamental sectors strictly outside itself, many points in $I_{0} \cup I_{2}$ have orbits that leave this set at some iteration. Let $\Gamma_{\lambda}$ be the set of points whose orbits remain for all iterations in $I_{0} \cup I_{2}$. Then we have:

Proposition. The set $\Gamma_{\lambda}$ is a Cantor set and $F_{\lambda} \mid \Gamma_{\lambda}$ is conjugate to the one-sided shift on two symbols.

Proof. By the previous result, each of the fundamental sectors is mapped in one-toone fashion onto the open region $\mathcal{O}$ that properly contains $I_{0} \cup I_{2}$ in $\mathbb{C}$. We therefore have a well-defined inverse $G_{0}$ (resp., $G_{2}$ ) of $F_{\lambda}$ that maps $\mathcal{O}$ onto $I_{0}$ (resp., $I_{2}$ ). Standard arguments then show that these inverses are contractions in the Poincaré metric on $\mathcal{O}$. Consequently, for any one-sided sequence $\left(s_{0} s_{1} s_{2} \ldots\right)$ of 0 's and 2's, the set

$$
\bigcap_{j=0}^{\infty} G_{s_{0}} \circ \ldots \circ G_{s_{j}}(\mathcal{O})
$$

is a unique point and the map that takes the sequence $\left(s_{0} s_{1} s_{2} \ldots\right)$ to this point defines a homeomorphism between the space of one-sided sequences of 0's and 2's endowed with the usual topology and $\Gamma_{\lambda}$. Hence $\Gamma_{\lambda}$ is a Cantor set and we have that $F_{\lambda} \mid \Gamma_{\lambda}$ is conjugate to the one-sided shift on two symbols. 
We remark that when $\lambda \in \mathbb{R}^{-}$, the Cantor set $\Gamma_{\lambda}$ lies on the real axis. Indeed, a glance at the graph of the real function $F_{\lambda}$ shows that $F_{\lambda}$ maps the interval $\left[-p_{\lambda}, p_{\lambda}\right]$ in two-to-one fashion over itself, where $p_{\lambda}$ is the fixed point for $F_{\lambda}$ on the positive real axis and on the boundary of $B_{\lambda}$. See Figure 4 . For complex values of $\lambda \in \mathcal{P}$ we let $p_{\lambda}$ be the fixed point lying in $I_{0}$, i.e., the point in $\Gamma_{\lambda}$ with itinerary $(000 \ldots)$.

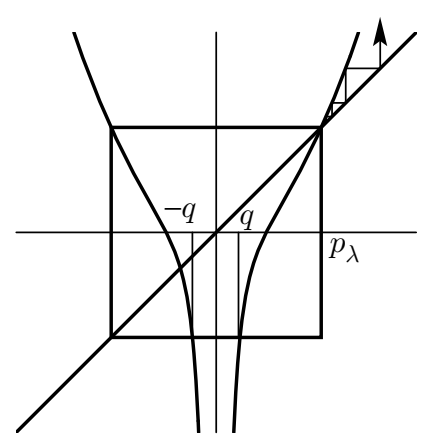

FiguRE 4 . The graph of $F_{\lambda}$ on the real line for $\lambda<0$. The points $\pm q$ bound the trap door on the real axis.

Note also that if $\lambda \in \mathcal{P}$, the Julia set of $F_{\lambda}$ need not be a connected set. Indeed, when $|\lambda|=1$ we have $\left|v_{\lambda}\right|=2$, so by the Escape Criterion, the critical orbits escape to $\infty$ and $J\left(F_{\lambda}\right)$ is a Cantor set. This also occurs for $\lambda \in \mathcal{P}$ with $|\lambda|$ close to 1 .

2.2. Internal rays. In this section, we first construct special internal rays of angles 0 and $1 / 2$ for parameters in $\mathcal{P}$. These internal rays will be continuous curves that pass from $\partial B_{\lambda}$ to $\partial T_{\lambda}$ (when $\lambda$ is in the connectedness locus) and contain the entire Cantor set $\Gamma_{\lambda}$ as well as countably many curves in preimages of $T_{\lambda}$.

Consider the closed sector $S_{0}=S_{0}(\lambda)$ bounded by the two rays $\theta=\eta / 4$ and $\theta=\eta / 4-\pi / 2$ where $\eta=\operatorname{Arg} \lambda$. These were the rays that formed the straight line boundaries of the smaller region $I_{0}$ in the previous section. Let $S_{2}=-S_{0}$. We include $\infty$ in both of these sectors. As in the case of $I_{0}$ and $I_{2}, F_{\lambda}$ maps the boundaries of these sectors strictly outside of the sectors (except at 0 and $\infty$ ). Now consider the set of points in $S_{0} \cup S_{2}$ whose entire orbits lie in $S_{0} \cup S_{2}$. Let $R_{0}=R_{0}^{\lambda}$ be the portion of this set that lies in $S_{0}$. We call $R_{0}$ the full ray of angle 0 . Similarly, let $R_{1 / 2}$, the full ray of angle $1 / 2$, be the corresponding set in $S_{2}$. Our next goal is to prove that $R_{0} \cup R_{1 / 2}$ is a simple closed curve in the Riemann sphere passing through both 0 and $\infty$ as well as the Cantor set $\Lambda_{\lambda} . R_{0}$ will also contain the external ray $\xi_{0}^{\lambda}$ as well as an analogous ray near 0 that maps to the external ray $\xi_{1 / 2}^{\lambda}$. The complement of these two rays in $R_{0}$ will be the internal ray of angle 0 .

Since we are considering parameters in the region $\mathcal{P}$, a certain amount of these parameters lie outside the connectedness locus. In this case it is not clear that the external rays are well defined since they may contain a critical point. However, we do have:

Proposition. Suppose $\lambda \in \mathcal{P}$. Then the external ray $\xi_{0}^{\lambda}(t)$ is defined for all $t \in$ $[0,1)$ and lies in the right half plane. This ray limits on $p_{\lambda}$ as $t \rightarrow 1$. 
Proof. Consider the portion of the sector $S_{0}$ that lies outside of the critical circle. Call this region $U_{0} . F_{\lambda}$ maps $U_{0}$ to a half plane whose boundary is the straight line that passes through the origin, both critical values, and $\infty$. Since $\lambda$ is not positive and real, it follows that $F_{\lambda}$ maps the boundary of $U_{0}$ strictly outside $U_{0}$ (except at $\infty)$. Note that the fixed point $p_{\lambda}$ lies in $U_{0}$.

Let $\omega_{0}$ be the set of points whose orbits are entirely contained in $U_{0}$. Since $U_{0}$ is connected and $F_{\lambda}\left(U_{0}\right) \supset U_{0}$, we have that $\omega_{0}$ is a connected set that contains $\infty$ and $p_{\lambda}$. This follows since the preimage of $U_{0}$ in $U_{0}$ is a closed, connected subset of $S_{0}$ containing these points, and so each successive preimage has this property. Therefore $\omega_{0}$ is connected.

For each $\lambda \in \mathcal{P}$, we have a Böttcher coordinate $\phi_{\lambda}$ defined at least in a neighborhood of $\infty$. Therefore the external ray $\xi_{0}^{\lambda}$ is defined in some neighborhood of $\infty$. Then, since $F_{\lambda}$ maps the boundary of $U_{0}$ strictly outside $U_{0}$, we must have that a portion of the curve $\xi_{0}^{\lambda}$ near $\infty$ resides inside $U_{0}$.

Now, if $\lambda$ lies in the connectedness locus, we know that the curve $\xi_{0}^{\lambda}$ connects $p_{\lambda}$ to $\infty$. But suppose that some portion of this curve does not lie in $U_{0}$. Let $z_{\lambda}$ be the point on this curve that lies in $\partial U_{0}-\infty$ and is the closest such point to $\infty$ on the curve $\xi_{0}^{\lambda}$ where closest means the nearest point to $\infty$ along the curve $\xi_{0}^{\lambda}$. Then we must have that $F_{\lambda}\left(z_{\lambda}\right)$ lies in the interior of $U_{0}$ since points on the invariant curve $\xi_{0}^{\lambda}$ move closer to $\infty$ under iteration of $F_{\lambda}$. But this contradicts the fact that $F_{\lambda}$ maps $\partial U_{0}-\infty$ outside $U_{0}$. Therefore we have $\xi_{0}^{\lambda} \subset \omega_{0}$ in this case.

In fact, we have $\xi_{0}^{\lambda} \cup p_{\lambda}=\omega_{0}$, for if there were some other point in $\omega_{0}$, then the orbit of this point would necessarily tend to $\infty$ since all points in $\Gamma_{\lambda} \cap U_{0}$ except $p_{\lambda}$ eventually enter $S_{2}$. But, near $\infty$, only points on the external ray $\xi_{0}^{\lambda}$ remain for all iterations in $U_{0}$. So we have $\xi_{0}^{\lambda} \cup p_{\lambda}=\omega_{0}$ when $\lambda$ lies in the connectedness locus.

If $\lambda$ does not lie in the connectedness locus, we can pull the locally defined portion of $\xi_{0}^{\lambda}$ near $\infty$ back by the inverse of $F_{\lambda}$ on $U_{0}$. As above, these pullbacks can never meet $\partial U_{0}$ or either of the critical values. Hence the complete external ray is well defined for all inverses of $F_{\lambda} \mid U_{0}$. This ray must then limit on some points in $I_{0} \cap U_{0}$ and these limit points must have orbits that remain in $I_{0}$ for all iterations. But the only point in $I_{0} \cap U_{0}$ that has this property is $p_{\lambda}$. Thus, in this case, we also have that the entire external ray of angle 0 is well defined and connects $p_{\lambda}$ and $\infty$, and so $\omega_{0}=\xi_{0}^{\lambda} \cup p_{\lambda}$ as before.

In a similar fashion, the external ray $\xi_{1 / 2}^{\lambda}(t)$ is defined for all $t \in[0,1)$ and $\lambda \in \mathcal{P}$. This ray lies in $S_{2}$ and limits on $-p_{\lambda}$ as $t \rightarrow 1$. Let $q_{\lambda}$ be the preimage of $-p_{\lambda}$ lying in $I_{0}$. Then $-q_{\lambda}$ is the other preimage of $-p_{\lambda}$ that lies in $I_{2}$. Then there is a curve $\tau_{\lambda}$ passing through the origin that is mapped two-to-one onto $\xi_{1 / 2}^{\lambda}$ by $F_{\lambda}$. This curve connects $\pm_{\lambda}$ and lies in $T_{\lambda}$ when $\lambda$ is in the connectedness locus. Let $\tau_{0}^{\lambda}$ (resp., $\tau_{1 / 2}^{\lambda}$ ) be the portion of $\tau_{\lambda}$ that connects 0 to $q_{\lambda}$ (resp., $-q_{\lambda}$ ). We may then parametrize both of these curves by simply pulling back the corresponding parametrization of $\xi_{1 / 2}^{\lambda}(t)$, i.e., $F_{\lambda}\left(\tau_{0}^{\lambda}(t)\right)=\xi_{1 / 2}^{\lambda}(t)$. We call these rays the trap door rays.

Proposition. The full ray $R_{0} \cup R_{1 / 2}$ is a simple closed curve lying in $S_{0} \cup S_{2}$ and passing through 0 and $\infty$.

Proof. Since $R_{0} \cup R_{1 / 2}$ is the set of points whose orbits remain for all iterations in the set $S_{0} \cup S_{2}$, this set certainly contains all of the preimages of the curve $\tau_{\lambda}$ whose forward orbits lie in $S_{0} \cup S_{2}$ until landing on $\tau_{\lambda}$. This set also includes the 
Cantor set $\Gamma_{\lambda}$. In fact, these two collections of points together with $\xi_{0}^{\lambda}$ and $\xi_{1 / 2}^{\lambda}$ are precisely the set $R_{0} \cup R_{1 / 2}$. This follows since any other such point cannot have an orbit that remains for all iterations in $I_{0} \cup I_{2}$, since $\Gamma_{\lambda}$ is exactly the set with this property. Therefore the orbit of such a point must leave this region. But then this orbit must tend to $\infty$, and hence the orbit must land on some external ray. But the only external rays that remain for all iterations in $S_{0} \cup S_{2}$ are the external rays of angles 0 and $1 / 2$.

We now show that the union of all of the preimages of $\tau_{\lambda}$ in $R_{0} \cup R_{1 / 2}$ together with the Cantor set $\Gamma_{\lambda}$ is a pair of continuous curves, one in $S_{0}$ and one in $S_{2}$. To see this, recall that the dynamics on $\Gamma_{\lambda}$ are given by the shift map on two symbols. We can then conjugate $F_{\lambda}$ on $\Gamma_{\lambda}$ to the piecewise linear map on the Cantor middle-thirds set given by

$$
L(x)= \begin{cases}3 x & \text { if } 0 \leq x \leq 1 / 2 \\ 3-3 x & \text { if } 1 / 2 \leq x \leq 1\end{cases}
$$

Let $h_{\lambda}$ be this conjugacy defined on the middle-thirds Cantor set. Here we assume that $h_{\lambda}(0)=p_{\lambda}$ and $h_{\lambda}(1)=-p_{\lambda}$ and the symbolic dynamics for $L$ on the Cantor middle-thirds set matches exactly with that for $F_{\lambda}$ on $\Gamma_{\lambda}$. We can then extend this map to a continuous map taking $(1 / 3,2 / 3)$ to the curve $\tau_{\lambda}$ with $h_{\lambda}(1 / 2)=0$. Taking appropriate pullbacks, we can then define $h_{\lambda}$ so that it takes each of the removed intervals in $[0,1]$ to the appropriate preimage of $\tau_{\lambda}$. This defines a map from the unit interval to $R_{0} \cup R_{1 / 2}$. So the question is whether or not $h_{\lambda}$ is continuous.

Suppose this is not the case. Since $h_{\lambda}$ is defined continuously on the removed open intervals, it must be the case that $h_{\lambda}$ is not continuous at some point $x$ in the Cantor middle-thirds set. Suppose that $h_{\lambda}(x)=z_{*}$. Then $z_{*}$ lies in the Cantor set $\Gamma_{\lambda}$. Thus there must be a sequence of points $x_{j}$ converging to $x$ for which $h_{\lambda}\left(x_{j}\right)$ converges to some point $w_{*} \neq z_{*}$. We may assume that the points in this sequence all lie in the complement of the Cantor middle-thirds set in $[0,1]$ since we know that $h_{\lambda}$ is continuous on the Cantor set. We may further assume that each $x_{j}$ lies in a distinct removed open interval.

Now we know that the endpoints of these removed open intervals in $[0,1]$ are mapped to a sequence of points in $\Gamma_{\lambda}$ that converge to $z_{*}$. Thus the images of the removed intervals must be mapped to curves that, in the limit, stretch from $z_{*}$ to $w_{*}$, i.e., have non-zero length. But we know that the map $F_{\lambda}$ is expanding on the set $I_{0} \cup I_{2}$. It follows that the pullbacks of the curve $\tau_{\lambda}$ must have lengths that tend to 0 . This gives a contradiction and shows that the full rays are indeed continuous curves.

Recall that $q_{\lambda} \in I_{0}$ is the point that is mapped by $F_{\lambda}$ to $-p_{\lambda}$ and that $-q_{\lambda}$ is the corresponding point in $I_{2}$. We now define the internal ray $\ell_{0}^{\lambda}$ to be the portion of $R_{0}$ lying in $S_{0}$ and connecting $p_{\lambda}$ to the point $q_{\lambda}$. Similarly, the internal ray $\ell_{1 / 2}^{\lambda}$ is the piece of $R_{1 / 2}$ connecting $-p_{\lambda}$ to $-q_{\lambda}$. Note that the internal rays $\ell_{0}^{\lambda}$ and $\ell_{1 / 2}^{\lambda}$ are each mapped by $F_{\lambda}$ one-to-one onto the union of these rays together with the curve $\tau_{\lambda}$ lying in $T_{\lambda}$.

Now recall that $F_{\lambda}$ also maps the sets $I_{1}$ and $I_{3}$ onto the set $F_{\lambda}\left(I_{0}\right)=F_{\lambda}\left(I_{2}\right)$. Hence there is a curve that is mapped onto $\ell_{0}^{\lambda} \cup \tau_{\lambda} \cup \ell_{1 / 2}^{\lambda}$ in each of these sets. These curves are the internal rays of angles $1 / 4$ (lying in $I_{1}$ ) and $3 / 4$ (in $I_{3}$ ) and we denote them as above by $\ell_{1 / 4}^{\lambda}$ and $\ell_{3 / 4}^{\lambda}$. See Figure 5. We define the full ray of 
angle $1 / 4, R_{1 / 4}^{\lambda}$, to be the preimage of $R_{0} \cup R_{1 / 2}$ that lies in the sector $S_{1}$ and let $R_{3 / 4}$ be the corresponding full ray lying in $S_{3}$.

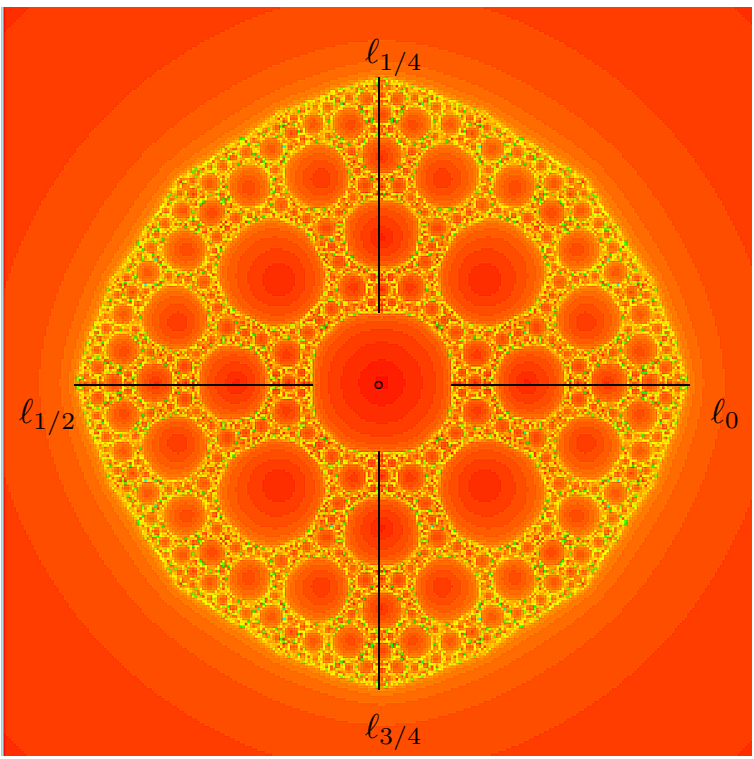

FiguRE 5. The Julia set and some internal rays for $\lambda=-1 / 16$. The principal Cantor necklace $N_{0}^{-1 / 16}$ straddles the real axis in this case.

For later use, all four of the internal rays $\ell_{j}^{\lambda}$ may be parametrized by $t \in[0,1]$ in the following way. For example, consider $\ell_{0}^{\lambda}$. First consider the Cantor set portion of this ray. The map $h_{\lambda}$ takes the portion of the Cantor middle-thirds set lying in $[0,1 / 3]$ to this set. Then, rescaling linearly by a factor of 3 , we define $\ell_{0}^{\lambda}(t)=h_{\lambda}(t / 3)$. This defines the parametrization of the Cantor set portion of this ray and we have that $\ell_{0}^{\lambda}(0)=p_{\lambda}$ and $\ell_{0}^{\lambda}(1)=q_{\lambda}$. Next, recall that we have parametrizations $\tau_{0}^{\lambda}(t)$ and $\tau_{1 / 2}^{\lambda}(t)$ of the trap door rays of angles 0 and $1 / 2$ in $T_{\lambda}$. Each is defined on the interval $[0,1)$. Then we may pull these parametrizations back in the natural way to the removed intervals by linearly rescaling the map so that the midpoints of these intervals are sent to the points that eventually map to 0 . Thus this parametrization is dynamically defined and depends continuously on $t$ and analytically on $\lambda$ since the Cantor set portion and the various preimages of $T_{\lambda}$ all depend analytically on $\lambda$.

To summarize:

Proposition. If $\lambda \in \mathcal{P}$, we have the four continuous internal rays $\ell_{j}^{\lambda}(t)$ where $j=0,1 / 4,1 / 2$, and $3 / 4$ and $t \in[0,1]$. When $\lambda$ lies in the connectedness locus, each of these rays extends from $\partial B_{\lambda}$ to $\partial T_{\lambda}$. For any given value of $t$, the map $\lambda \mapsto \ell_{j}^{\lambda}(t)$ is analytic in $\lambda$.

2.3. Cantor necklaces in the dynamical plane. To define a Cantor necklace, let $M$ denote the Cantor middle-thirds set in the unit interval $[0,1]$. For each open interval of length $1 / 3^{n}$ removed from the unit interval in the construction of $M$, 
we replace this interval by an open disk of diameter $1 / 3^{n}$ whose closure meets the Cantor middle-thirds set only at the endpoints of the removed interval. We call the resulting set in the plane the Cantor middle-thirds necklace. See Figure 6. Any set that is the image of Cantor middle-thirds necklace under a continuous, one-to-one map is then called a Cantor necklace.

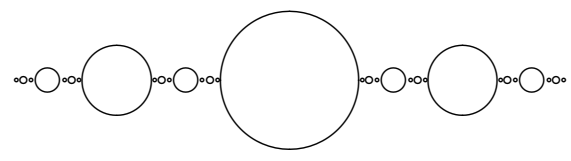

Figure 6. The Cantor middle-thirds necklace.

Remark. We do not include the boundary of the open regions in a Cantor necklace for the following technical reason: it is sometimes difficult in practice to verify that these bounding curves of Cantor necklaces are simple closed curves. Also, we do not require that the map taking the Cantor middle-thirds necklace to its image be a homeomorphism since sequences of the open sets in the Cantor necklace could theoretically accumulate in some complicated fashion on points in the Cantor set that are not endpoints (though we know of no specific case in the family $F_{\lambda}$ where this actually occurs).

Now suppose that $\lambda \in \mathcal{P}$ lies in the connectedness locus in the parameter plane. Thus $T_{\lambda}$ is disjoint from $B_{\lambda}$. The principal Cantor necklace for $F_{\lambda}, N_{0}^{\lambda}$, is then the union of the Cantor set $\Gamma_{\lambda}$ together with $T_{\lambda}$ and all of its preimages that meet the internal rays $\ell_{0}^{\lambda}$ and $\ell_{1 / 2}^{\lambda}$. That is, $N_{0}^{\lambda}$ simply enlarges the internal rays $\ell_{0}^{\lambda}$ and $\ell_{1 / 2}^{\lambda}$ by adjoining $T_{\lambda}$ and its appropriate preimages to these arcs. Since there are no critical points in any of these preimages of $T_{\lambda}$, we have a Böttcher coordinate on each such disk. Rescaling the Böttcher coordinates linearly to each preimage of $T_{\lambda}$, we get a homeomorphism taking each preimage of $T_{\lambda}$ to the appropriately sized disk in the Cantor middle-thirds necklace. Thus we have:

Theorem. Suppose that $\lambda$ lies in the connectedness locus and that $0<\operatorname{Arg} \lambda<2 \pi$. Then the set $\Gamma_{\lambda}$ together with the appropriate preimages of $T_{\lambda}$ forms a Cantor necklace.

See Figure 5 for an example of the principal Cantor necklace for a parameter $\lambda \in \mathbb{R}^{-}$.

Remarks. (1) $F_{\lambda}$ maps $N_{0}^{\lambda}$ two-to-one over itself and $B_{\lambda}$.

(2) There is also a pair of similar invariant Cantor necklaces defined for $\lambda$ positive real and in the connectedness locus, though we will not deal with this case in this paper. See [8].

\section{Cantor necklaces in the Parameter Plane}

In this section we show that there is an analogue of the principal Cantor necklace as well as the corresponding internal rays in the parameter plane and that this necklace straddles the negative real axis. 
3.1. The principal necklace. Recall that $\mathcal{P}$ is the open unit disk in the parameter plane with the non-negative real axis removed, so $\mathcal{P}$ is a simply connected set in $\mathbb{C}$. For each $\lambda \in \mathcal{P}$, we have the internal ray of angle $1 / 4$ in the dynamical plane given by $\ell_{1 / 4}^{\lambda}(t)$ where $t \in[0,1]$. This ray is contained in the region $I_{1}$ for all $\lambda \in \mathcal{P}$, and so it always lies within the half-disk $D$ given by $|z|<2$ and $\operatorname{Im}(\mathrm{z})>0$. So for each $t \in[0,1]$, the map $\lambda \mapsto \ell_{1 / 4}^{\lambda}(t)$ is an analytic function that maps $\mathcal{P}$ inside $D$. We have another map that takes $\mathcal{P}$ onto $D$, namely the map $V(\lambda)=v_{\lambda}=2 \sqrt{\lambda}$ where $v_{\lambda}$ is the critical value lying in the upper half plane. Note that $V$ is invertible, so, for fixed $t$, we can consider the composition $G_{t}(\lambda)=V^{-1}\left(\ell_{1 / 4}^{\lambda}(t)\right)$. The map $G_{t}$ is an analytic map that takes the simply connected region $\mathcal{P}$ inside itself, i.e., $G_{\lambda}: \mathcal{P} \rightarrow \mathcal{P}$. By the Schwarz Lemma, we know that if $G_{t}$ has a fixed point in $\mathcal{P}$, then that fixed point is unique. Furthermore, this fixed point varies continuously with $t$.

Let us assume for the moment that there is such a fixed point for $G_{t}$ (this may not happen since it could be the case that the fixed point lies in the boundary of $\mathcal{P}$ where these maps are not well-defined). Call this fixed point/parameter value $\lambda_{t}$. We have that $\lambda_{t}$ varies continuously with $t$. Then we know that the critical value of $F_{\lambda_{t}}$ in the upper half plane lands on the point $\ell_{1 / 4}^{\lambda_{t}}(t)$. Then $\lambda_{t}$ defines an internal ray in the parameter plane since there is a unique $t$-value corresponding to any given point in the Cantor set portion of the necklace as well as to all of the appropriate preimages of the pullbacks of the external rays. That is, there is a Cantor set of points on the curve $\lambda_{t}$ for which the orbit of the critical points of $F_{\lambda_{t}}$ does not escape to $\infty$, and the complement of this Cantor set in $\lambda_{t}$ consists of parameters for which the orbit of the critical points does escape. Note that, in the latter case, it takes at least two iterations for the critical orbits to enter the trap door, so, assuming that $\lambda_{t}$ is in the connectedness locus, maps with these parameters have Sierpiński curve Julia sets.

We can now adjoin open disks to these open intervals in the curve $\lambda_{t}$ to create a Cantor necklace in the parameter plane. It is known by a work of Roesch [20] that the set of parameters for which the critical point lies in a given preimage of $B_{\lambda}$ forms a simply connected open subset of the parameter plane. We call such a set a Sierpinski hole. It is also known that there is a unique parameter in each Sierpiński hole for which the orbit of the critical points all eventually land on 0 and then map to $\infty$; we call this parameter the center of the Sierpiński hole. All other parameters in this hole have the property that the orbit of the critical points tend to $\infty$ rather than land on $\infty$. Hence if we append any such Sierpiński hole to the corresponding escaping intervals in $\lambda_{t}$, just as we did in the dynamical plane construction, we see that this new set again has the structure of a Cantor necklace. This is the principal Cantor necklace in the parameter plane and we denote it by $\mathcal{C}$.

The only question that remains is: Does the map $G_{t}$ actually have a fixed point in the region $\mathcal{P}$ for each $t$. In fact, this is always the case except when $t=0$. If $t=0$, we have the situation where the critical value would lie on the first preimage of $p_{\lambda}$ in $\ell_{1 / 4}^{\lambda}$. This cannot happen, as we shall show later.

Now assume that $t>0$. If $\lambda \in \mathbb{R}^{-}$, then the graph of $F_{\lambda}$ (Figure 4) shows that the entire dynamical Cantor set $\Gamma_{\lambda}$ lies in $\mathbb{R}$ and we know that the internal rays of angles 0 and $1 / 2$ also lie in $\mathbb{R}$. Consider the map $S(\lambda)=F_{\lambda}^{2}\left(c_{\lambda}\right)=4 \lambda+1 / 4$, i.e., the second iterate of the critical points. Note that if $S(\lambda)$ lies on a point in 
the principal Cantor set in the dynamical plane, then a critical value must lie in the corresponding point in the $1 / 4$ ray, so this would yield a fixed point for our map $G_{t}$. Now when $\lambda \in \mathbb{R}^{-}, S(\lambda)$ is also real. When $\lambda=-1, S(\lambda)=-3.75$, and, consequently, $S(-1)$ lies well to the left of the point $-p_{\lambda}$. Therefore $S(-1)$ lies to the left of internal rays of angles 0 and $1 / 2$ when $\lambda=-1$. But as $\lambda \rightarrow 0$, $S(\lambda) \rightarrow 1 / 4$. This means that, for $\lambda$ close to $0, S(\lambda)$ now lies to the right of the internal ray of angle $1 / 2$, which lies in $\mathbb{R}^{-}$. Since the points on this ray vary continuously with $t$ and $\lambda$, it follows that there is indeed a point where $S(\lambda)$ lands on a point of the form $\ell_{1 / 2}(t)$ for any given $t \in[0,1]$.

Now consider a point in $\ell_{0}^{\lambda}(t)$. If this point is in the Cantor set portion of the internal ray, then we may associate an itinerary $s=\left(s_{0} s_{1} s_{2} \ldots\right) \in \Sigma_{2}$ to this point. Since this point lies in $I_{0}$ we have $s_{0}=0$. But since we are assuming that this point is not equal to $p_{\lambda}$, there must be a first $n$ for which $s_{n}=2$, i.e., $s=\left(0 \ldots 0 s_{n} \ldots\right)$ where $s_{n}=2$. For this point, the orbit of the corresponding point lies on the positive real axis for the first $n-1$ iterations, but then moves to $\mathbb{R}^{-}$at iteration $n$. Now if $\lambda$ is very small and negative, we have $F_{\lambda} \approx z^{2}$. Under $z^{2}$ the point $1 / 4$ has an orbit that remains for all iterations in $\mathbb{R}^{+}$. Therefore we can choose $\lambda$ small enough so that the point $1 / 4$ as well as $F_{\lambda}^{2}\left(c_{\lambda}\right) \approx 1 / 4$ have orbits that also remain in $\mathbb{R}^{+}$for more than $n$ iterations. For this $\lambda$-value, we then have that $S(\lambda) \approx 1 / 4>\ell_{0}^{\lambda}(t)$. Hence there must have been a $\lambda$-value somewhere between -1 and this value for which $S(\lambda)$ does indeed lie on the point with this itinerary. This says that the corresponding map $G_{t}$ does indeed have a fixed point if $t>0$.

When $t=0$ the point $\ell_{0}^{\lambda}(0)$ in $I_{0}$ is $p_{\lambda}$ and the graph of $F_{\lambda}$ shows that $p_{\lambda}>1 / 4$ for all parameters in $\mathcal{P} \cap \mathbb{R}^{-}$, so the above argument fails when $t=0$.

Since the points in the complement of the Cantor set portion on this ray (including the curve $\tau_{\lambda}$ ) move in a similar fashion, we also have a parameter for which $S(\lambda)$ lands on one of these points. This shows that we have a fixed point for $G_{t}$ for each $t>0$. Thus there is a principal Cantor necklace $\mathcal{C}$ in the parameter plane that straddles $\mathbb{R}^{-}$. (Technically, we should add in the origin to this set so that we have the full Cantor set portion of this necklace.)

The central Sierpiński hole in $\mathcal{C}$ now contains parameters for which the second iterate of the critical points lies in $T_{\lambda}$, so this is a Sierpiński hole with escape time 3. One checks easily that the center of this Sierpiński hole lies at $\lambda=-1 / 16$. We denote this hole by $\mathcal{S}_{3}$. We may then define the analogue of internal rays in the parameter plane as follows. The internal ray of angle 0 in the parameter plane, $\nu_{0}$, is the closed interval extending from 0 to the first point in $\mathbb{R}^{-}$lying in $\partial \mathcal{S}_{3}$. Also, the internal ray of angle $1 / 2, \nu_{1 / 2}$, extends from the other point in the boundary of $\mathcal{S}_{3}$ in $\mathbb{R}^{-}$all the way down to the last point in the connectedness locus in $\mathbb{R}^{-}$, i.e., the parameter for which the second iterate of the critical points all land on $-p_{\lambda}$.

We therefore have shown:

Theorem. There is a Cantor necklace $\mathcal{C}$ in the parameter plane whose Cantor set portion lies along the negative real axis and has the following properties:

(1) Any point in the Cantor set portion of $\mathcal{C}$ (except 0) is a parameter value $\lambda_{s}$ for which $F_{\lambda_{s}}^{2}\left(c_{\lambda_{s}}\right)$ has itinerary $s$ in $\Gamma_{\lambda_{s}}$.

(2) Any point in the complementary open regions of $\mathcal{C}$ are $\lambda$-values for which the critical orbit eventually lands in $B_{\lambda}$, so $J\left(F_{\lambda}\right)$ is a Sierpinski curve. 
(3) Any endpoint $\lambda_{s}$ of the Cantor set portion of $\mathcal{C}$ (except 0) is a parameter value for which the critical orbit eventually lands on the fixed point $p_{\lambda_{s}}$ in the boundary of $B_{\lambda_{s}}$.

We define the Cantor necklace $\mathcal{C}$ to be the "trunk" of the CMS tree. We display in Figure 7 the parameter plane for the family $F_{\lambda}$. The grey disks in this picture are the Sierpiński holes where the critical orbit eventually enters the trap door and so the Julia set is a Sierpiński curve. The largest grey region is the Sierpiński hole $\mathcal{S}_{3}$ with center at $-1 / 16$.

Remark. If $\lambda$ is a parameter that is a buried point in the Cantor set portion of the necklace in the parameter plane, then it is known that $J\left(F_{\lambda}\right)$ is a Sierpiński curve [4. However, if $\lambda$ is an endpoint in this Cantor set, then $J\left(F_{\lambda}\right)$ is a "hybrid" Sierpiński curve. That is, there are countably many pairs of preimages of $T_{\lambda}$ whose boundary curves touch each other at a single point. See [5].

3.2. Two other internal rays in the parameter plane. We now produce two other internal rays in the parameter plane $\nu_{\alpha}(t)$, where $\alpha=1 / 4$ and $\alpha=3 / 4$ together with the corresponding Cantor necklaces. The ray $\nu_{\alpha}$ will be defined to be the set of parameters for which the second iterate of the critical point, $F_{\lambda}^{2}\left(c_{\lambda}\right)$, lands on a point in the dynamical plane ray given by $\ell_{\alpha}^{\lambda}$.

First, consider the case $\alpha=1 / 4$. As we have shown, the internal ray $\ell_{1 / 4}^{\lambda}(t)$ in the dynamical plane lies in the region $I_{1}$ and is mapped one-to-one onto $\ell_{0}^{\lambda} \cup \tau_{\lambda} \cup \ell_{1 / 2}^{\lambda}$. For each $\lambda \in \mathcal{P}$, the region $I_{1}$ lies in the half-disk $D$ given by $\operatorname{Im} z>0$ and $|z|<2$. Also, as usual, for fixed $t, \ell_{1 / 4}^{\lambda}(t)$ varies analytically with $\lambda$ in this region. Thus the map $t \mapsto \ell_{1 / 4}^{\lambda}(t)$ maps $\mathcal{P}$ inside the half-disk $D$ and depends analytically on $\lambda$ for each fixed value of $t$.

Now the map $S(\lambda)=F_{\lambda}^{2}\left(c_{\lambda}\right)=4 \lambda+1 / 4$ is also defined on $\mathcal{P}$ and is invertible. Also, $S(\mathcal{P})$ contains the half-disk $D$, but not strictly, as the portion of the real line given by $[1 / 4,2]$ now lies in the boundary of the image of $S(\mathcal{P})$ and $D$. So we have one of the following: Either the analytic map $S^{-1}\left(\ell_{1 / 4}^{\lambda}(t)\right)$ has a unique fixed point in $\mathcal{P}$, or else the map has a fixed point on the common boundary of the images, namely the interval $[1 / 4,2]$. We claim that this latter case cannot occur. Suppose it does. Then $\lambda$ must be positive and real. Also, $\ell_{1 / 4}^{\lambda}(t)$ must either be in one of the preimages of the trap door or else be a point in the Cantor set portion of $\ell_{1 / 4}^{\lambda}$. But the graph of $F_{\lambda}$ shows that, when $\lambda \in \mathbb{R}^{+}$, there are no preimages of the trap door in $\mathbb{R}^{+}$(except $T_{\lambda}$, which is not part of $\ell_{1 / 4}^{\lambda}$ ). Hence $F_{\lambda}^{2}\left(c_{\lambda}\right)$ cannot land on a point on the real line in $\ell_{1 / 4}^{\lambda}$ that escapes to $\infty$. Also, if $\ell_{1 / 4}^{\lambda}(t)$ lies in the Cantor set portion of the necklace and $\mathbb{R}^{+}$, then the graph of $F_{\lambda}$ for positive $\lambda$ shows that the forward orbit of this point must remain forever on the positive real axis. But the only points in the Cantor set portion of the ray for which this could happen would be $p_{\lambda}$. Then, if $F_{\lambda}^{2}\left(c_{\lambda}\right)$ lands on $p_{\lambda}$, we would have that $v_{\lambda}$ lies on the boundary of the trap door. Hence we have nearby parameters for which the critical values lie in $T_{\lambda}$. But this cannot happen for this family of maps [14. This proves the existence of a curve of points in the parameter plane for which the second iterate of the critical points lands on $\ell_{1 / 4}^{\lambda}(t)$ and hence defines $\nu_{1 / 4}^{\lambda}(t)$. Adjoining the appropriate Sierpiński holes then defines the Cantor 1/4-necklace in the parameter plane. The Cantor 3/4-necklace given by $\nu_{3 / 4}$ is just the complex conjugate of this necklace. 
See Figure 7 for a picture of these Cantor necklaces. These two necklaces are the main branches in the CMS tree.

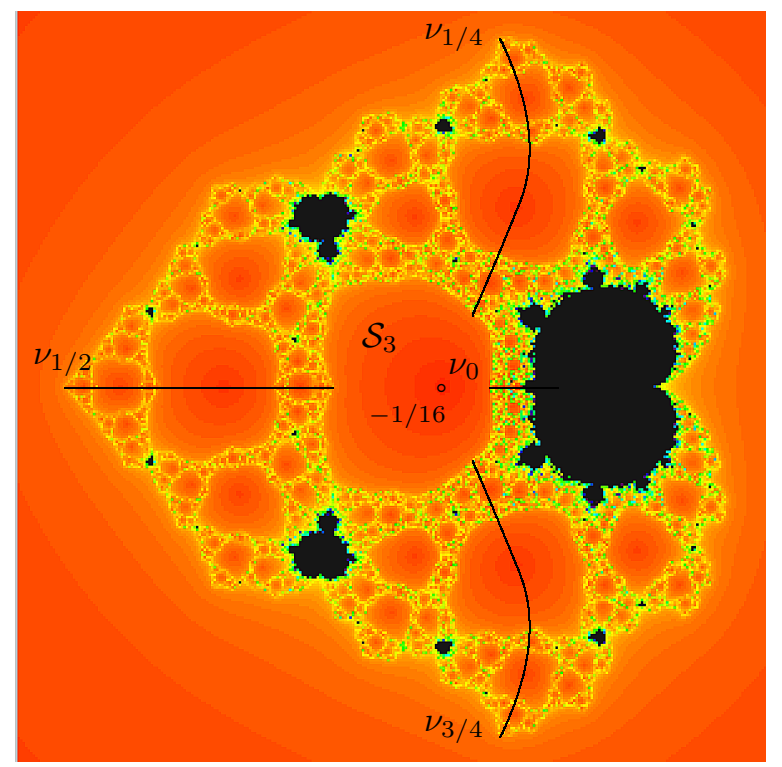

FiguRE 7. Some internal rays in the parameter plane.

\section{Construction of the CMS tree}

To construct the remaining branches of the CMS tree in the parameter plane, we first need to construct the analogues of these Cantor necklaces in the dynamical plane. By the $z \mapsto \bar{z}$ symmetry in the parameter plane, it will suffice to consider only the case where $0<\operatorname{Arg} \lambda<\pi$ in this section.

4.1. Location of the rays $R_{0}$ and $R_{1 / 2}$. In order to construct the CMS tree, we first need to show the following: When $0<\operatorname{Arg} \lambda<\pi$, the full ray $R_{0}$ in the dynamical plane lies below the real axis, while the full ray $R_{1 / 2}$ lies above the real axis. To show this, we will modify the construction of the Cantor set portion of the principal Cantor necklace by choosing smaller regions in which they reside.

There are two cases. First assume that $0<\operatorname{Arg} \lambda \leq 2 \pi / 3$. Earlier our region $I_{0}$ was contained in the sector bounded by the lines passing through $c_{\lambda}$ and $-i c_{\lambda}$. Now we modify this sector so that it is bounded by $\mathbb{R}^{+}$and the line through $-i c_{\lambda}$. Call this closed sector $J_{0}$. Let $J_{2}=-J_{0}$.

Proposition. Assuming $0<\operatorname{Arg} \lambda \leq 2 \pi / 3$, then $F_{\lambda}$ maps the boundaries of $J_{0}$ and $J_{2}$ strictly outside $J_{0} \cup J_{2}$ except at 0 and $\infty$.

Proof. First note that, since $0<\operatorname{Arg} \lambda \leq 2 \pi / 3$, we have $\operatorname{Arg} \lambda \leq \operatorname{Arg} c_{\lambda}+\pi / 2=$ $(\operatorname{Arg} \lambda) / 4+\pi / 2$. The image of the boundary curve $\mathbb{R}$ of $J_{0} \cup J_{2}$ is given by the curve $t \mapsto t^{2}+\lambda / t^{2}$ where $t \in \mathbb{R}$. Assuming that $t \neq 0$, we have that $t^{2} \in \mathbb{R}^{+}$and that $\lambda / t^{2}$ lies on the straight line extending from 0 to $\infty$ and passing through $\lambda$. The sum of these two points therefore is strictly contained in the sector $0<\operatorname{Arg} z<\operatorname{Arg} \lambda$, 
so this entire curve is strictly contained in this region that lies outside $J_{0} \cup J_{2}$. As earlier, the image of the other boundary curve of the sectors, namely the line passing through the critical points $\pm i c_{\lambda}$, is the ray extending from the critical value to $\infty$ in the lower half plane. This line also lies outside $J_{0} \cup J_{2}$.

As a consequence of this Proposition, the full ray $R_{0}$ (resp., $R_{1 / 2}$ ) lies in the sector $J_{0}$ (resp., $J_{2}$ ) when $0<\operatorname{Arg} \lambda \leq 2 \pi / 3$ and hence below (resp., above) $\mathbb{R}^{+}$.

Now consider the case $2 \pi / 3<\operatorname{Arg} \lambda<\pi$. We will now modify the sectors $J_{0}$ and $J_{2}$ as follows. One boundary curve will still be $\mathbb{R}$, but the other will now be the straight line given by $t \lambda$ where $t \in \mathbb{R}$. Then $J_{0}$ is defined by $-\operatorname{Arg} \lambda \leq \operatorname{Arg} z \leq 0$ and $J_{2}=-J_{0}$.

Proposition. When $2 \pi / 3<\operatorname{Arg} \lambda<\pi$, we again have that $F_{\lambda}$ maps the boundaries of $J_{0}$ and $J_{2}$ strictly outside $J_{0} \cup J_{2}$ except at 0 and $\infty$.

Proof. As in the previous Proposition, $F_{\lambda}$ maps $\mathbb{R}$ to a curve that resides in the interior of the region $0<\operatorname{Arg} z<\operatorname{Arg} \lambda$, so this curve lies outside $J_{0} \cup J_{2}$. Then the line $-t \lambda$ for $t \in \mathbb{R}$ is mapped to the curve $t^{2} \lambda^{2}+1 /\left(t^{2} \lambda\right)$. Therefore the point $t^{2} \lambda^{2}$ lies on a line that is strictly contained in the sector $-\pi<\operatorname{Arg} z<-\operatorname{Arg} \lambda$ and hence outside $J_{0} \cup J_{2}$. Similarly, the point $1 /\left(t^{2} \lambda\right)$ lies on the ray passing through $1 / \lambda$ which, by assumption, lies in the sector $-\pi<\operatorname{Arg} z<-2 \pi / 3$. Again the sum of points on the image of this line therefore lies outside the region $J_{0} \cup J_{2}$.

It therefore follows from these two Propositions that, if $0<\operatorname{Arg} \lambda<\pi$, the full ray $R_{1 / 2}^{\lambda}$ lies in the upper half plane when $0<\operatorname{Arg} \lambda<\pi$, while $R_{0}^{\lambda}$ lies in the lower half plane. By symmetry, $R_{1 / 4}$ lies in the right half plane, while $R_{3 / 4}$ lies in the left half plane for these parameter values. Given the existence of these four full rays, we may now partition the dynamical plane into four more dynamically defined sectors. From now on, we let $S_{0}=S_{0}^{\lambda}$ be the closed region bounded by the full rays $R_{0}$ and $R_{1 / 4}$. Let $S_{j}$ for $j=1,2,3$ be the corresponding sectors arranged in the counterclockwise direction. Note that $F_{\lambda}$ maps each of $S_{0}$ and $S_{2}$ two-to-one onto $S_{0} \cup S_{1}$, while $S_{1}$ and $S_{3}$ are each mapped two-to-one onto $S_{2} \cup S_{3}$. Also, each $S_{j}$ contains a unique critical point with $c_{\lambda}=\lambda^{1 / 4}$ lying in $S_{0}$.

Proposition. Suppose $\lambda$ lies in the portion of $\mathcal{P}$ in the upper half plane. Then the critical value $v_{\lambda}$ lying in the upper half plane always lies in the interior of $S_{0}$ while the other critical value lies in the interior of $S_{2}$.

Proof. If this were not the case, since $F_{\lambda}\left(S_{0}\right)=S_{0} \cup S_{1}$, we must have either $v_{\lambda} \in R_{1 / 4}$ or else $v_{\lambda} \in S_{1}$. But we know that the only parameters in $\mathcal{P}$ for which $v_{\lambda}$ lies in $R_{1 / 4}$ are those along $\mathbb{R}^{-}$, so this cannot happen. Now, when $\lambda \in \mathbb{R}^{+}$, the critical value $v_{\lambda} \in \mathbb{R}^{+}$. Therefore $v_{\lambda}$ certainly lies in $S_{0}$ for parameters close to the positive real axis. If there were some other parameter for which $v_{\lambda}$ lies in $S_{1}$, then a curve in $\mathcal{P}$ connecting this parameter to $\mathbb{R}^{+}$would necessarily pass through a parameter for which $v_{\lambda}$ lies in $R_{1 / 4}$, so this situation also cannot occur. Therefore $v_{\lambda} \in S_{0}$ for all such parameters in the upper half plane. By the $z \mapsto-z$ symmetry, the other critical value lies in $S_{2}$.

4.2. Other internal rays and Cantor necklaces. In this section, we pull back the previously defined internal rays to construct infinitely many other such objects in the dynamical and parameter planes. We shall now assume that $\lambda$ lies in the 
portion of $\mathcal{P}$ that lies in the upper half plane. Call this set $\mathcal{P}^{+}$. Thus we have $|\lambda|<1$ and $0<\operatorname{Arg} \lambda<\pi$. Since $\lambda \in \mathcal{P}^{+}$, by the previous Proposition, the two critical values lie in the regions $S_{0}$ and $S_{2}$. It follows that there are a pair of disjoint subsectors in the region $S_{3}$ that are mapped one-to-one onto $S_{3}$, while the complement of these subsectors is a connected set that contains the unique critical point in $S_{3}$ and is mapped two-to-one onto $S_{2}$. One of the subsectors mapped to $S_{3}$ meets the full ray $R_{0}$ along the portion of this ray that extends from $\infty$ to the center of the preimage in $\ell_{0}^{\lambda}$ of the trap door; the other meets the full ray $R_{3 / 4}$ in a similar curve that now extends from $\ell_{3 / 4}^{\lambda}$ to 0 , not $\infty$. These two subsectors are symmetric under the involution $H_{\lambda}$. We consider only the preimage of $S_{3}$ that meets $R_{0}$. Call this subsector $T$.

Since $T$ is mapped one-to-one onto $S_{3}$, there is a preimage of the ray $R_{3 / 4}$ that lies in the boundary of $T$. We call this curve the full ray with angle $7 / 8, R_{7 / 8}$. This ray extends from the prepole lying in $R_{0}$ to $\infty$. Since $T$ is mapped one-to-one over itself, there is a preimage of $R_{7 / 8}$ lying in $T$; this is the ray $R_{15 / 16}$. This ray extends from the unique preimage of the above prepole in $R_{0}$ to $\infty$. Continuing in this fashion, we find an infinite collection of rays $R_{1-1 / 2^{n}}$, one for each $n \geq 3$. These rays are all disjoint and each is mapped one-to-one onto $R_{3 / 4}$ by $F_{\lambda}^{n-2}$ and extends from a particular preimage of the prepole in $R_{0}$ to $\infty$.

Now consider the sector $S_{1}$. By the $z \mapsto-z$ symmetry, we have a similar collection of rays given by $-R_{1-1 / 2^{n}}$. These are the full rays whose angles are $1 / 2-1 / 2^{n}$. These rays now extend from $R_{1 / 2}$ to $\infty$, and they are also mapped univalently onto $R_{3 / 4}$ by $F_{\lambda}^{n-2}$. Let $\gamma_{n}^{\lambda}$ be the portion of the full ray $R_{1 / 2-1 / 2^{n}}$ that is mapped by $F_{\lambda}^{n-2}$ onto the portion of $R_{3 / 4}$ that contains both the trap door ray and internal ray, i.e., $R_{3 / 4}-\xi_{3 / 4}^{\lambda}$. As earlier, we have a natural parametrization $\gamma_{n}^{\lambda}(t)$ obtained by pulling back the parametrization of $R_{3 / 4}$. We may also enlarge these curves to form Cantor necklaces (where we exclude the preimages of the trap door that lies in the ray $R_{1 / 2}$ ). In a similar fashion, using the fourfold symmetry, we have rays of angle $1 / 4-1 / 2^{n}$ in the sector $S_{0}$ and angle $3 / 4-1 / 2^{n}$ in the sector $S_{2}$.

We may now construct the analogous rays in the parameter plane just as we did earlier. Each curve $\gamma_{n}^{\lambda}(t)$ is strictly contained in the upper half plane. Thus we may again consider, for given $t$, the map $\lambda \mapsto S^{-1}\left(\gamma_{n}^{\lambda}(t)\right)$ where $S$ is the second iterate of the critical point. This map is defined on the disk $\mathcal{P}^{+}$which is mapped by $S$ strictly over the set containing $\gamma_{n}^{\lambda}(t)$. Unlike the previous cases, for each such $t$, there is then a unique fixed point for this map. This is a parameter $\lambda_{n}^{t}$ for which the second iterate of the critical point lands on the corresponding point in $\gamma_{n}^{\lambda}(t)$. As before, the curve $t \mapsto \lambda_{n}^{t}$ depends continuously on $t$ and contains a Cantor set of parameters that land in the Cantor set portion of $\gamma_{n}^{\lambda}(t)$ as well as countably many open intervals that lie in Sierpiński holes. Note that this curve extends outward from the principal Cantor necklace in the parameter plane to the Cantor set locus, and each is disjoint from all the others. Denote these curves in the parameter plane by $\nu_{\alpha_{n}}(t)$, where $\alpha_{n}=1 / 2-1 / 2^{n}$. Then $\nu_{\alpha_{n}}$ is the internal ray in the parameter plane with angle $\alpha_{n}$. As before we can again expand these curves to Cantor necklaces by adding in the entire Sierpiński hole around each such open interval. 
By the $z \mapsto \bar{z}$ symmetry in the parameter plane, we have a similar collection of internal rays lying in the lower half plane. These are the internal rays with angles $1 / 2+1 / 2^{n}$. We have shown:

Theorem. There are infinitely many disjoint Cantor necklaces in the parameter plane corresponding to the angles $\beta_{n}=1 / 2 \pm 1 / 2^{n}$. Each branches off the principal Cantor necklace and extends to the boundary of the Cantor locus in the parameter plane.

See Figure 8 for a picture of some of these Cantor necklaces. Adjoining these Cantor necklaces to the principal Cantor necklace in the parameter plane yields the Cantor necklace structure of the CMS tree.

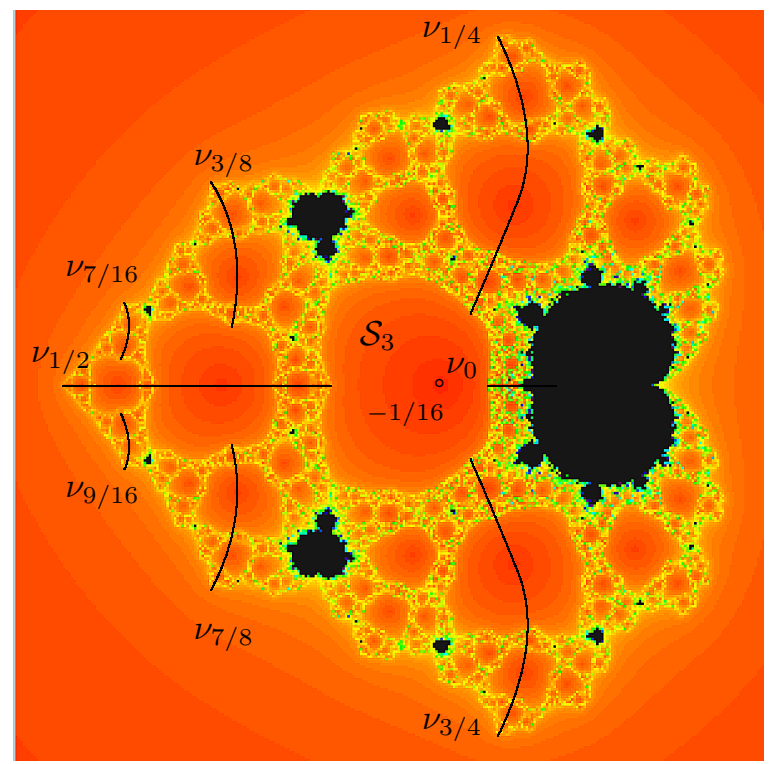

FiguRE 8. More Cantor necklaces in the parameter plane.

Remark. In this paper we have considered only the special internal rays of angles $1 / 2 \pm 1 / 2^{n}$. In fact, one can pull back the internal rays of angles 0 and $1 / 2$ to construct internal rays of angles $p / 2^{n}$ for any positive integers $p$ and $n$. See [2]. A natural open question is then: What about other internal rays? These have yet to be investigated.

4.3. Baby Mandelbrot sets in the CMS tree. We now prove the existence of infinitely many Mandelbrot sets in the parameter plane for $F_{\lambda}$, one between each pair of branches in the CMS tree.

The main tool that we shall use is that of polynomial-like maps (see [13]). This tool allows us to find baby Mandelbrot sets provided that the following is true. Suppose we have a family of maps $f_{\lambda}: U_{\lambda} \rightarrow V_{\lambda}$ where $\lambda$ is contained in a disk $\mathcal{O}$ in some parameter plane and

- $f_{\lambda}, U_{\lambda}$, and $V_{\lambda}$ all vary analytically with $\lambda$;

- $U_{\lambda}$ and $V_{\lambda}$ are bounded, simply connected regions in the plane; 
- $U_{\lambda} \subset V_{\lambda}$ for each $\lambda \in \mathcal{O}$;

- $f_{\lambda}: U_{\lambda} \rightarrow V_{\lambda}$ is proper of degree two.

Such a family of maps is called a polynomial-like family of maps of degree two. Suppose further that, as $\lambda$ runs clockwise around the boundary of $\mathcal{O}$, the unique critical value of $f_{\lambda}$ circles clockwise around the annular region $V_{\lambda}-U_{\lambda}$. Then it is known that the set of parameters $\lambda$ for which the critical orbit of $f_{\lambda}$ remains for all iterations in $U_{\lambda}$ is a copy of the Mandelbrot set and, moreover, the set of points whose orbit remains in $U_{\lambda}$ for such a $\lambda$ determines a set homeomorphic to the filled Julia set of the corresponding quadratic polynomial in the actual Mandelbrot set [13.

Using this polynomial-like map tool, we first produce the large Mandelbrot set lying in the region between the rays $R_{1 / 4}$ and $R_{7 / 8}$ and bounded below by $R_{1 / 2}$. This is a Mandelbrot set whose main cardioid contains parameters with an attracting two-cycle. We therefore say that this Mandelbrot set has base period two. Let the disk $\mathcal{O}$ in the parameter plane be our half-disk $\mathcal{P}^{+}$. For $\lambda \in \mathcal{P}^{+}$, let $U_{\lambda}$ be the "triangular" region bounded by the rays $R_{3 / 4}, R_{5 / 8}$, and the circle $|z|=2$. This disk is mapped one-to-one by $F_{\lambda}$ onto a disk that is bounded by the rays $R_{1 / 4}, R_{1 / 2}$, and the image of the circle $|z|>2$, namely $\beta_{\lambda}$, that again lies strictly outside $|z|=2$. Then $F_{\lambda}$ maps $F_{\lambda}\left(U_{\lambda}\right)$ two-to-one onto a disk $V_{\lambda}$ that is bounded by $R_{1 / 2}$ and a curve in the half plane below $R_{1 / 2}$ that lies strictly outside $\beta_{\lambda}$ and so outside $|z|=2$. We then choose our polynomial-like map $f_{\lambda}$ to be $F_{\lambda}^{2}$, which therefore maps $U_{\lambda}$ two-to-one onto the disk $V_{\lambda}$ that strictly contains $U_{\lambda}$. Thus, for each $\lambda \in \mathcal{P}^{+}, f_{\lambda}: U_{\lambda} \rightarrow V_{\lambda}$ is polynomial-like of degree two.

Now, as $\lambda$ rotates around the boundary of $\mathcal{P}^{+}$, the critical value of $F_{\lambda}$ lying in the lower half plane rotates once around the boundary of the quarter-disk $|z|=$ $2, \operatorname{Re} z, \operatorname{Im} \mathrm{z} \leq 0$. We claim that this disk strictly contains $U_{\lambda}$ for each $\lambda \in \mathcal{P}^{+}$ since $R_{5 / 8}$ lies in the lower half plane.

Lemma. For $\lambda \in \mathcal{P}^{+}$, the ray $R_{5 / 8}$ lies in the region $\operatorname{Re} z, \operatorname{Im} z<0$.

Proof. Let $X$ be the region bounded by $R_{1 / 2}$ and the negative real axis, where we recall that, by the results in Section 4.1 , for $\lambda \in \mathcal{P}^{+}, R_{1 / 2}$ always lies in the upper half plane. Then $F_{\lambda}$ maps $X$ one-to-one onto a region bounded below by $R_{0} \cup R_{1 / 2}$ and above by the curve $t \mapsto t^{2}+\lambda / t^{2}$ where $t \in \mathbb{R}^{-}$. Since there are no critical points in $X$, there are no critical values in $F_{\lambda}(X)$. Hence the critical value $v_{\lambda}$ in the sector $S_{0}$ lies above $F_{\lambda}(X)$.

Choose a curve $\zeta$ connecting $v_{\lambda}$ to $\infty$ in $S_{0}$ and lying outside of $F_{\lambda}(X)$. Then the preimage of $\zeta$ in $S_{2}$ is a curve joining 0 to $\infty$ and passing through the critical point. By construction, $F_{\lambda}^{-1}(\zeta)$ does not meet $\mathbb{R}^{-}$.

Now there are two preimages of $S_{1}$ in $S_{2}$ and each is disjoint from $F_{\lambda}^{-1}(\zeta)$. One of these preimages is the region between $R_{5 / 8}$ and $R_{3 / 4}$. Since $R_{3 / 4}$ lies below $F_{\lambda}^{-1}(\zeta)$, so too must $R_{5 / 8}$. Therefore $R_{5 / 8}$ lies in the region $\operatorname{Re} z, \operatorname{Im} z<0$.

By the results in 13, we therefore have a baby Mandelbrot set in the parameter plane corresponding to values of $\lambda$ for which orbits of $F_{\lambda}$ bounce back and forth repeatedly between $U_{\lambda}$ and the sector $S_{1}$.

For the other baby Mandelbrot sets, the construction is a little different. Recall that we have the rays $R_{\alpha_{n}}$ where the angle $\alpha_{n}=1 / 2-1 / 2^{n}$ for $n \geq 3$ that lie in the sector $S_{1}$. Let $A_{n}$ be the "rectangle" in $S_{1}$ bounded by the rays of angle $\alpha_{n}$ and $\alpha_{n+1}$ together with a piece of the ray $R_{1 / 2}$ and the circle $|z|=2$. 
By the $z \mapsto-z$ symmetry, we have similar "rectangles" in the sector $S_{3}$. Let $B_{n}=-A_{n}$ for $n \geq 3$. Then $B_{n}$ is bounded by the rays of angle $\gamma_{n}=1-1 / 2^{n}$ and $\gamma_{n+1}$ as well as $R_{0}$ and the circle of radius 2. Let $B_{2}$ be the portion of the sector $S_{3}$ that is the complement of $\cup B_{n}$ inside the circle of radius 2 . Therefore the union of all of the $B_{n}$ fills the portion of $S_{3}$ lying inside the circle of radius 2. Now for each $n \geq 3$, we have that $F_{\lambda}$ maps $B_{n}$ univalently over $B_{n-1}$. The boundaries of $B_{n}$ lying in $R_{0}$ are mapped to the corresponding boundary of $B_{n-1}$. The boundaries lying in the rays $R_{\gamma_{j}}$ are mapped into a slightly larger portion of $R_{\gamma_{j-1}}$. Also, the outer circular boundary of $B_{n}$ is mapped to the curve $\beta_{\lambda}$ that lies outside $|z|=2$. Let $C_{3}$ be the preimage of $B_{2}$ lying in $B_{3}$. Then $F_{\lambda}$ now maps $C_{3}$ univalently onto $B_{2}$. Inductively, let $C_{j+1}$ be the preimage of $C_{j}$ lying in $B_{j+1}$. Then $F_{\lambda}^{j-2}\left(C_{j}\right)=B_{2}$.

The set $B_{2}$ is a little different from the other $B_{j}$. This set contains the critical point that lies in sector $S_{3}$ and is mapped two-to-one over a set containing the portion of the sector $S_{2}$ lying inside $|z|=2$. $B_{2}$ also contains the sets $H_{\lambda}\left(B_{n}\right)$ for each $n \geq 3$, so $F_{\lambda}$ also maps $B_{2}$ one-to-one over all of the other $B_{n}$, though this is not important in the polynomial-like map construction below.

Now we have that $F_{\lambda}$ maps the rectangle $A_{n}$ lying in the sector $S_{1}$ univalently over $B_{n-1}$ for each $n \geq 3$. Let $\tilde{A}_{n}$ be the subset of the rectangle $A_{n}$ that is mapped univalently onto $C_{n-1}$.

The critical point that lies in the sector $S_{2}$ is mapped to the critical value that lies in $S_{0}$ by our assumption that $\lambda \in \mathcal{P}^{+}$. Hence there is a pair of preimages of each $\tilde{A}_{n}$ in the portion of the sector $S_{2}$ contained within the circle of radius 2 . One of these preimages has boundary that meets the ray $R_{3 / 4}$, while the other preimage is symmetrically located with respect to $H_{\lambda}$ and meets the ray $R_{1 / 2}$. Let $\tilde{U}_{n}$ be the preimage of $\tilde{A}_{n}$ that meets the ray $R_{3 / 4}$. Thus $F_{\lambda}: \tilde{U}_{n} \rightarrow \tilde{A}_{n}$ is one-to-one and onto. See Figure 9 for a caricature of the $A_{n}, B_{n}$, and $U_{n}$.

Now we have that $\tilde{U}_{n}$ is mapped one-to-one onto $\tilde{A}_{n}$. Then $\tilde{A}_{n}$ is mapped oneto-one onto $C_{n-1}$, which is mapped one-to-one onto $C_{n-2}$, etc. Thus we have that $F_{\lambda}^{n-1}$ maps $\tilde{U}_{n}$ univalently onto the region $B_{2}$. But then $F_{\lambda}$ maps $B_{2}$ two-to-one over a region that contains the set $\tilde{U}_{n}$. Hence $F_{\lambda}^{n}$ is a polynomial-like map of degree 2 on $\tilde{U}_{n}$. Note that the critical value of $F_{\lambda}^{n}$ is just the critical value of $F_{\lambda}$ that lies in the lower half plane (each iterate of $F_{\lambda}$ is univalent except for the last iterate defined on $B_{2}$ ). We claim that, just as in the first case, as $\lambda$ rotates around the boundary of $\mathcal{P}^{+}$, the critical value of $F_{\lambda}^{n}$ circles around in the complement of $\tilde{U}_{n}$ in $F_{\lambda}^{n}\left(\tilde{U}_{n}\right)=F_{\lambda}\left(B_{2}\right)$. This follows since $\tilde{U}_{n}$ is contained inside the region bounded by $R_{5 / 8}, R_{3 / 4}$, and the circle of radius 2 which, by the previous Lemma, lies inside the region $\operatorname{Re} z, \operatorname{Im} z<0$. Consequently, the critical value of $F_{\lambda}^{n}$ rotates similarly in $S_{2}$ with regard to the $\tilde{U}_{n}$. This produces a baby Mandelbrot set of base period $n$ for each $n \geq 3$. 


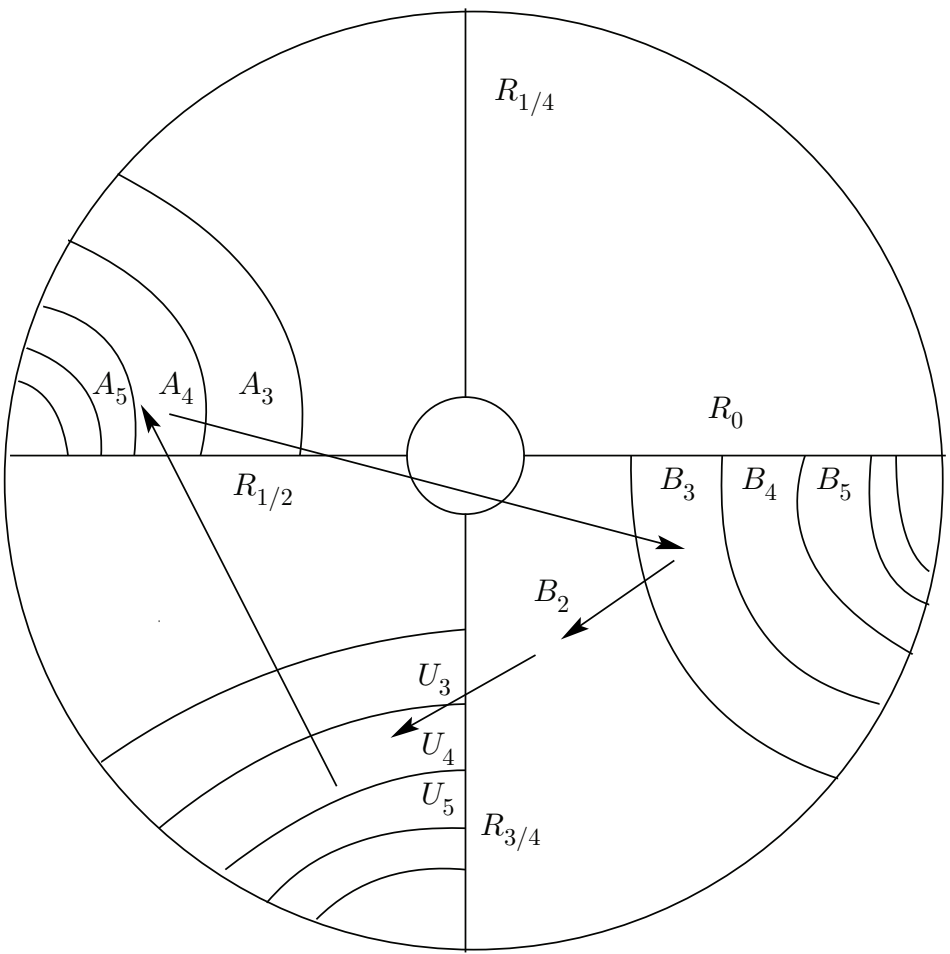

Figure 9. The $A_{n}, B_{n}$, and $U_{n}$ and an orbit drawn from a parameter in the baby Mandelbrot set of base period 4 .

\section{REFERENCES}

[1] Blanchard, P., Devaney, R. L., Look, D. M., Seal, P., and Shapiro, Y. Sierpiński Curve Julia Sets and Singular Perturbations of Complex Polynomials. Ergodic Theory and Dynamical Systems. 25 (2005), 1047-1055. MR2158396 (2006d:37087)

[2] Çilingir, F., Devaney, R. L., Russell, E. D. Extending External Rays Throughout the Julia Sets of Rational Maps. J. Fixed Point Theory and Applications 7 (2010), 223-240. MR:2652518 (2012g:37091)

[3] Devaney, R. L., Fagella, N., Garijo, A., and Jarque, X. Sierpiński Curve Julia Sets for Quadratic Rational Maps. To appear.

[4] Devaney, R. L. and Look, D. M. A Criterion for Sierpiński Curve Julia Sets. Topology Proceedings 30 (2006), 163-179. MR2280665 (2007j:37071)

[5] Devaney, R. L. and Look, D. M. Buried Sierpiński Curve Julia Sets. Discrete and Continuous Dynamical Systems 13 (2005), 1035-1046. MR2166716(2006d:37088)

[6] Devaney, R. L., Look, D. M., and Uminsky, D. The Escape Trichotomy for Singularly Perturbed Rational Maps. Indiana University Math. J. 54 (2005), 1621-1634. MR2189680 (2006i:37105)

[7] Devaney, R. L. and Pilgrim, K. Dynamic Classification of Escape Time Sierpiński Curve Julia Sets. Fundamenta Mathematicae 202 (2009), 181-198. MR2506193 (2010f:37081)

[8] Devaney, R. L. Cantor Necklaces and Structurally Unstable Sierpiński Curve Julia Sets for Rational Maps. Qualitative Theory of Dynamical Systems 2 (2004), 337-359. MR 2275444 (2007i:37091)

[9] Devaney, R. L. Intertwined Internal Rays in Julia Sets of Rational Maps. Fundamenta Mathematicae 206 (2009), 139-159. MR2576265 (2011f:37078)

[10] Devaney, R. L. Dynamics of $z^{n}+\lambda / z^{n} ;$ Why the Case $n=2$ is Crazy. In Conformal Dynamics and Hyperbolic Geometry. Contemporary Math. AMS 573 (2012), 49-65. 
[11] Devaney, R. L. Cantor Webs in the Parameter and Dynamical Planes of Rational Maps. Fields Institute Communications 53 (2008), 105-123. MR:2477420 (2009m:37130)

[12] Devaney, R. L. The McMullen Domain: Satellite Mandelbrot Sets and Sierpiński Holes. Conformal Geometry and Dynamics 11 (2007), 164-190. MR2346215 (2008f:37106)

[13] Douady, A. and Hubbard, J. Itération des Polynômes quadratiques complexes. C.R. Acad. Sci. Paris, t.29, Serie I-1982, pp. 123-126. MR651802 (83m:58046)

[14] McMullen, C. Automorphisms of Rational Maps. Holomorphic Functions and Moduli. Vol. 1. Math. Sci. Res. Inst. Publ. 10. Springer, New York, 1988. MR955807 (89m:58187)

[15] McMullen, C. The Classification of Conformal Dynamical Systems. Current Developments in Mathematics. Internat. Press, Cambridge, MA, 1995, 323-360. MR1474980 (98h:58162)

[16] Milnor, J. Dynamics in One Complex Variable. Vieweg, 1999. MR.1721240 (2002i:37057)

[17] Milnor, J. and Tan Lei. A "Sierpiński Carpet" as Julia Set. Appendix F in Geometry and Dynamics of Quadratic Rational Maps. Experiment. Math. 2 (1993), 37-83. MR.1246482 (96b:58094)

[18] Morosawa, S. Local Connectedness of Julia Sets for Transcendental Entire Functions. In Proc. Int'l. Conf. on Nonlinear Analysis and Convex Analysis. World Scientific (1999), 266273. MR1742079 (2000m:37074)

[19] Petersen, C. and Ryd, G. Convergence of Rational Rays in Parameter Spaces. In The Mandelbrot Set: Theme and Variations. ed. Tan Lei. Cambridge Univ. Press (2000), 161-172. MR:1765088 (2001f:37057)

[20] Roesch, P. On Captures for the Family $f_{\lambda}(z)=z^{2}+\lambda / z^{2}$. In Dynamics on the Riemann Sphere, ed., P. Horth and C. Petersen. European Math Society, 2006. MR2348958 (2008j:37101)

[21] Steinmetz, N. Sierpiński and non-Sierpiński curve Julia sets in Families of Rational Maps. J. London Math. Soc. 3 (2008), 290-304. MR2439626 (2009h:37094)

[22] Sullivan, D. Quasiconformal Maps and Dynamical Systems I, Solutions of the Fatou-Julia Problem on Wandering Domains. Ann. Math. 122 (1985), 401-418. MR819553 (87i:58103)

[23] Whyburn, G. T. Topological Characterization of the Sierpiński Curve. Fund. Math. 45 (1958), 320-324. MR0099638(20:6077)

Department of Mathematics, Boston University, 111 Cummington Mall, Boston, MasSACHUSETTS 02215 